\title{
Endeudamiento y salud: dos conceptos relacionados en el desarrollo del pensamiento so- cioeconómico
}

\section{Rosalía de la Vega Guzmán ${ }^{1}$ y Rocio Zariñana Herrejón ${ }^{1}$}

\section{Resumen}

El endeudamiento es una condición generada a través del acceso a bienes y servicios por medio del crédito. En la cultura del endeudamiento están involucrados factores psicológicos y cognitivos que comprometen el bienestar físico y mental de las personas, por lo tanto, el comportamiento económico estará influido por la comprensión del entorno socioeconómico, así como por las habilidades, actitudes y motivaciones hacia la deuda, entre otras. El objetivo de este trabajo es conocer los significados asociados al concepto de endeuda-

1 Universidad Michoacana de San Nicolás de Hidalgo, rosaliadelavega@gmail.com 
miento durante el fin de semana del "Buen fin" de 2017, en población general de la ciudad de Morelia, Michoacán. Se realizó un estudio descriptivo a partir de la aplicación de redes semánticas naturales sobre el concepto de endeudamiento, con 39 participantes de entre 19 y 75 años de edad. Se obtuvieron 113 palabras definitorias de las cuales 9 tuvieron el mayor peso semántico, 7 de ellas hacen referencia a aspectos financieros y 2 a aspectos psicológicos relacionados con la salud. Las reflexiones finales apuntan a que las concepciones del endeudamiento están posiblemente influenciadas por las experiencias derivadas del comportamiento económico y sus consecuencias. En este contexto, predomina la relación entre lo financiero y la salud.

Palabras clave: psicología económica; endeudamiento; comportamiento económico; alfabetización económica; salud.

\section{Abstract}

Being in debt is a condition generated through access to goods and services by means of credit. In the culture of indebtedness, psychological and cognitive factors are involved that compromise the physical and mental wellbeing of people, therefore, economic behavior will be influenced by the understanding of the socioeconomic environment, as well as by skills, attitudes and motivations towards debt, among other. The aim of this work is to find the meanings associated with the concept of indebtedness during the "Buen Fin" weekend of 2017, in 
the general population of the city of Morelia, Michoacán. A descriptive study was carried out based on the application of natural semantic networks on the concept of indebtedness, with 39 participants between 19 and 75 years of age. 113 defining words were obtained, of which 9 had the highest semantic weight, 7 of them refer to financial aspects and 2 to psychological aspects related to health. Final reflections suggest that the concepts of indebtedness are possibly influenced by the experiences derived from economic behavior and its consequences. In this context, the relationship between finances and health predominates.

Keywords: economic psychology; indebtedness; economic behavior; economic literacy; health.

La relación entre el endeudamiento y la salud pareciera ser un tema del dominio público, es decir, la población en general podría deducir el efecto de uno sobre el otro sin ser especialistas en economía ni en medicina o psicología, sin embargo es necesario indagar más sobre la forma en que el estado económico de los seres en sociedad puede repercutir en el estado de sa-lud, específicamente en lo referente al endeudamiento.

Respecto al endeudamiento en México, puede advertirse un aumento en los índices de la cartera venci$\mathrm{da}$, los cuales se elevaron en un $12 \%$ con respecto a los datos registrados en los últimos tres años de acuer- 
do al análisis que hacen especialistas financieros, es decir, llegó a 38 mil 116 millones de pesos en enero de 2017. Este fenómeno puede ser el reflejo del incremento de las tasas de interés en 2016 por el Banco de México, que afectó los créditos al consumo (Esquivel, 2017). Respecto a lo anterior, existen opiniones a favor del endeudamiento, como la de Ernesto Torres Cantú, director de Citibanamex, quien con base en el fenómeno del aumento de la contratación de tarjetas de crédito durante el primer semestre de 2017, afirma que esta condición favorece las prácticas de consumo, considerando que este hecho es una muy buena noticia para la economía (Hernández, 2017). Como sea, estos datos permiten reflexionar sobre los efectos del acceso al crédito en los bolsillos de los ciudadanos y en sus repercusiones, ya que si los recursos económicos con los que cuentan no son suficientes para cubrir los gastos ordinarios, recurrir al crédito como una manera de solucionar situaciones económicas difíciles, puede convertirse en una situación de endeudamiento con diversas afecta-ciones y consecuencias (Denegri et al., 2016; Blázquez y Budría, 2015).

Por otra parte, existe evidencia que respalda la relación entre el nivel de endeudamiento y la falta de habilidades para el manejo de los recursos económicos y financieros (Denegri et al., 2016; Lusardi y Tufano, 2015), así como con la escasa información sobre el funcionamiento de las instituciones financieras (DiezMartínez, 2009). Además se considera que, más allá de 
las cuestiones financieras, acceder al crédito sin contar con una adecuada alfabetización económica también puede tener efectos en el bienestar físico y mental de las personas (Reis, Mendes y Grigion, 2015).

Pero ¿cómo se comprende el comportamiento económico de aquellos ciudadanos que se enfrentan a una sociedad económica compleja? Algunas de estas cuestiones han sido abordadas por la psicología económica, que estudia el comportamiento humano en relación con aspectos económicos y su percepción (Van Raaij, 1981) lo cual es útil para responder al objetivo de este estudio. Hoy en día la psicología económica, parece cobrar importancia ante las implica-ciones que tienen los fenómenos económicos en una variedad de aspectos, entre ellos la salud física y mental de las personas (Blázquez y Budría, 2015; Downing, 2016; Falconi et al., 2016; Shen, Sam y Jones, 2014).

Una de las líneas de interés de la psicología económica se centra en la alfabetización económica, definida como la adquisición de conocimientos, desa-rrollo de capacidades, actitudes y comportamientos relacionados con la obtención, uso y administración de recursos económicos, lo cual permite la comprensión del entorno socioeconómico y facilita la toma de deci-siones y el bienestar del individuo (Danes, 1994; Denegri et al., 2013; Diez-Martínez, 2009; Guth, Wärneryd y Lea, 1992). Para Van Raaij (1999) el comportamiento de las personas como consumidores y ciudadanos implica decisiones económicas y sus consecuencias. Por lo tanto, 
en el comportamiento económico siempre está presente el manejo de habilidades relacionadas con la toma de decisiones económicas. En este sentido, la solicitud de crédito y el uso del mismo como una extensión de los ingresos, sumados a una baja alfabetización económica y finan-ciera, puede incrementar el riesgo de contraer un estado de sobre-endeudamiento con implicaciones eco-nómicas, familiares, sociales y de salud (Denegri et al., 2013).

El endeudamiento se entiende como el gasto que va más allá de los ingresos y que reduce las ganancias por ser un consumo diferido, es decir, la utilización del bien es previa al consumo o pago del mismo (Katona, 1951). Desde este enfoque, la condición de endeudamiento puede estar determinada por varias situaciones, entre ellas: altas expectativas en el estilo de vida con bajos ingresos (Katona y Mueller, 1995), conductas materialistas, compras compulsivas, uso excesivo de la tarjeta de crédito y escases de alfabetización econó-mica (Reis et al., 2015), así como conductas que pueden generar una alta tolerancia al endeudamiento (Denegri et al., 2016; Mansilla, Denegri y Álvarez, 2016).

Desde otro punto de vista, una actitud hacia el endeudamiento, implica aspectos cognitivos -conocimiento de efectos y consecuencias: privación económica, conflictos legales-, afectivos —emociones y sentimientos ante la deuda: malestar personal, familiar, económico, social y de salud-y conductuales - acciones para salir de la deuda- (Lozano y Fuentes, 2011). 
Estas actitudes pueden estar determinadas por la evitación o tolerancia al uso cotidiano del crédito, dando como resultado actitudes de tipo austero o hedonista (Denegri et al., 2017). Es así que, para una persona con actitudes hedonistas, el hecho de renun-ciar a un deseo tiene implicaciones emocionales de represión o escasez de libertad, debido a que en su pensamiento económico existen valores prevalecientes y un modelo socioeconómico en donde el bienestar se interpreta como la satisfacción de los deseos a través del dinero y las deudas (Quintanilla, 2010). Sin embargo, existen estudios que demuestran que algu-nas consecuencias de la deuda se ven reflejadas en un bajo nivel de bienestar financiero (Reis et al., 2015; ), en la presencia de emociones negativas (Donnelly, lyerb y Howell, 2012), en manifestaciones de estrés (Durante y Laran, 2016; Dunn y Mirzaie, 2016) y en afectaciones a la salud mental (Clayton, Liñares-Zegarra y Wilson, 2015).

Hay evidencia que demuestra que en personas de menores recursos se dan mayores niveles de acceso a servicios de instituciones denominadas micro financieras, como las instituciones de crédito que son creadas por tiendas comerciales o de conveniencia. Ello les permite solventar determinadas situaciones económi-cas, conllevando un alto riesgo de sobre-endeudamiento (Angulo, 2014). En el caso de México, el llamado "Buen fin" podría ser un ejemplo de las condiciones que promueven el estado de sobre-endeudamiento a través de comportamientos económicos perjudiciales con ina-de- 
cuadas decisiones (García, 2017), resultados contrarios a los que plantean las políticas públicas generadas por los gobiernos actuales con la llamada "Inclusión financiera" (Comisión Nacional Bancaria y de Valores, 2017). Respondiendo a este escenario, el presente estudio tiene como objetivo conocer los significados asociados al concepto de endeudamiento durante el fin de semana del "Buen fin" de 2017, en población gene-ral de la ciudad de Morelia, Michoacán.

\section{Método}

Se realizó un estudio descriptivo utilizando la técnica de redes semánticas naturales para indagar las palabras asociadas al concepto de endeudamiento (Valdez, 1996). El estudio se realizó con población general del municipio de Morelia, Mich., en centros comerciales durante el fin de semana del "Buen fin" 2017.

\section{Participantes}

La selección de los participantes fue al azar empleándose un muestreo no probabilístico intencional. La muestra estuvo conformada por 39 personas, de las cuales 21 son mujeres (53.85\%) y 18 son hombres (46.15\%), con edades de los 19 a los 75 años ( $M=$ 45.6 , DE = 8.5), 21 de ellos solteros $(53.85 \%)$ y $17 \mathrm{ca}$ sados (43.58\%). En cuanto a la escolaridad de los participantes, 4 de ellos habían completado secundaria 
(10.25\%), 5 contaban con preparatoria (12.82\%), 4 tenían nivel técnico (10.25\%), 15 habían completado una licenciatura (38.46\%) y 8 contaban con estudios de posgrado (20.51\%). Del resto, 3, no se contó con información en esta variable (7.71\%). El único criterio de inclusión utilizado fue la disposición de los partici-pantes para llenar el formato descrito a continuación.

\section{Instrumento}

Se utilizó un formato que incluía una ficha de identificación con datos sociodemográficos (edad, sexo, estado civil, escolaridad) y un espacio para escribir las palabras asociadas al término de endeudamiento.

\section{Procedimiento}

La recolección de los datos se realizó afuera de centros comerciales de la ciudad de Morelia, Michoacán, en México durante dos de los cuatro días del "Buen fin" del año 2017. A las personas que aceptaron participar voluntariamente se les pidió que escribieran 10 palabras que para ellos definieran el concepto de endeudamiento, sin considerar artículos ni proposi-ciones. Posteriormente, se les pidió que jerarquizaran en orden de importancia cada una de las palabras, es decir, debían poner un 10 a la palabra que definiera más el concepto y así sucesivamente hasta poner 1 a la que menos defi- 
niera el concepto. El tiempo de aplicación aproximado por participante fue de 15 minutos.

Análisis de los datos

Los datos obtenidos se vaciaron y se procesaron en una hoja de cálculo de Excel, de acuerdo con los siguientes pasos: (1) se vaciaron todas las palabras escritas por los participantes; (2) se registró el nivel jerárquico asignado a cada una de las palabras, (3) se identificó el valor $\mathrm{J}$, es decir, el total de palabras definitorias expuestas por los participantes (riqueza semántica); y posteriormente (4) se calculó el valor $M$, que considera el peso semántico de cada palabra definitoria (Valdez, 1996; Hinojosa, 2008). Una vez realizado este procedimiento, se eligieron las palabras definitorias con mayor peso semántico mencionadas por los participantes, las cuales hacen referencia al significado que tiene el concepto (Valdez, 1996; Hinojosa, 2008).

Resultados

Los resultados de la investigación mostraron un valor J de 113 palabras definitorias, es decir, riqueza semántica de la red o tamaño de la red (Hinojosa, 2008; Reyes, 1993). Del total de palabras definitorias, 9 tuvieron el mayor peso semántico. En la Tabla 1 se observan los 
resultados de las palabras con mayor valor $M$ (peso semántico de cada palabra definidora), el valor FMG (porcentaje que indica la distancia semán- tica de cada concepto respecto al concepto principal de la red), las frecuencias respectivas y el porcentaje de los participantes que eligieron cada palabra.

Tabla 1.

Valores de la red semántica del concepto de endeudamiento

\begin{tabular}{lrrrc}
\hline Palabra definitoria & \multicolumn{1}{c}{ M } & FMG & \multicolumn{1}{c}{$f$} & $\%$ \\
\hline Deuda & 144 & $100 \%$ & 26 & 66.66 \\
Tarjeta de crédito & 94 & $65 \%$ & 12 & 30.76 \\
Prestamista & 79 & $55 \%$ & 15 & 38.46 \\
Prestado & 78 & $54 \%$ & 9 & 23.07 \\
Banco & 60 & $42 \%$ & 9 & 23.07 \\
Crédito & 56 & $39 \%$ & 12 & 30.76 \\
Estrés & 54 & $37 \%$ & 9 & 23.07 \\
Hipoteca & 53 & $37 \%$ & 8 & 20.51 \\
Angustia & 50 & $35 \%$ & 9 & 23.07 \\
\hline
\end{tabular}

En la Figura 1, se muestra el conjunto SAM, resultado del total de la población. En él se observa que el concepto principal de la red semántica es deuda, seguido de tarjeta de crédito, prestamista, prestado, banco, crédito, estrés, hipoteca y angustia. 


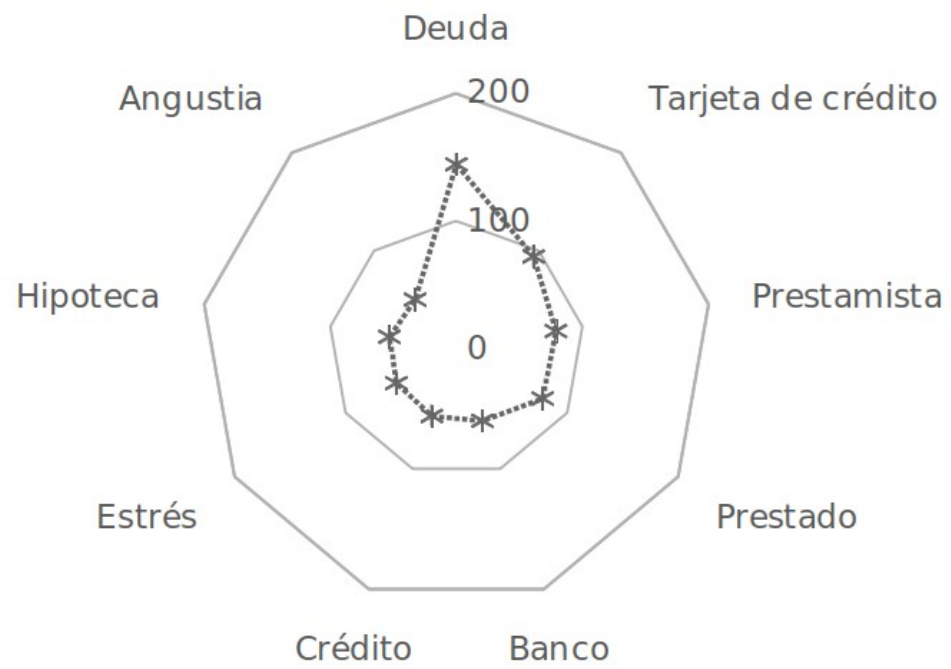

Figura 1.

Red semántica natural total: representación de endeudamiento.

\section{Discusión y conclusiones}

El estudio realizado permitió conocer los significados asociados al concepto de endeudamiento durante el fin de semana del "Buen fin" con población general de la ciudad de Morelia, Michoacán. Se observa que de las 9 palabras definitorias con mayor peso semántico, 7 de ellas hacen referencia a aspectos financieros (deuda, tarjeta de crédito, prestamista, prestado, banco, crédito, hipoteca) y las otras 2 tienen relación con la salud mental (angustia, estrés), estando relacionadas con aspectos psicológicos. Es relevante señalar que una de cada tres personas mencionó las palabras estrés y angustia 
respecto al concepto de endeudamiento. Estos aspectos están relacionados a la salud mental, con afecciones que pueden ser identificadas fácilmente y que repercuten en el bienestar general de las personas (Downing, 2016; Shen et al., 2014). Estos resultados son congruentes con algunos estudios que determinan que el endeudamiento es una condición que favorece el deterioro de la salud mental (Clayton et al., 2015; Durante y Laran, 2016).

Los resultados obtenidos llevan a la reflexión de que las concepciones acerca del concepto de endeudamiento están posiblemente influenciadas por experiencias, aprendizajes y consecuencias derivados de la práctica cotidiana de los sujetos económicos y de su nivel de alfabetización económica y financiera, en donde se pone de manifiesto la toma de decisiones y los aspectos cognitivos por medio de los cuales se da un acercamiento a la comprensión del complejo mundo socioeconómico que nos rodea (Delval, 2007; Diez-Martínez, 2009; Denegri, Sepulveda y Layera, 2018).

En este contexto, es evidente que la concepción del endeudamiento está ligada a dos principales aspectos: lo financiero (deuda, tarjeta de crédito, prestamista, prestado, banco, crédito, hipoteca) y la salud mental (angustia y estrés), estos hallazgos son compatibles con otros estudios que identifican el impacto del endeudamiento sobre el estado de salud y bienestar en las personas (Blázquez y Budría, 2015; Durante y Laran, 2016; Dunn y Mirzaie, 2016; Clayton et al., 2015; Dow- 
ning, 2016; Falconi et al., 2016; Reis et al., 2015; Shen et al., 2014)

Cabe mencionar que existieron algunas limita-ciones para la realización del estudio, entre ellas de tipo metodológico, pues se contó con poca partici-pación para contestar el formulario. A pesar de lo anterior, el presente estudio pretende contribuir a las investigaciones de la psicología económica a través de la aproximación al concepto de endeudamiento dentro del marco contextual de un evento socioeconómico de consumo, apoyado y derivado de una política pública sustentada y justificada en el interés de la derrama económica, pero con repercusiones en el bolsillo de los ciudadanos, ya que favorece al estado de endeuda-miento a largo plazo.

Con recomendaciones para futuras investiga-ciones, se sugiere ampliar la muestra de participantes y explorar otros conceptos derivados del estudio del comportamiento económico como el del ahorro, la inversión, entre otros. Además sería conveniente considerar las condiciones sociodemográficas (edad, nivel socioeconómico, capital cultural, género, nivel educativo, entre otros) para obtener más detalles sobre las posibles diferencias sociales, contextuales y de género.

\section{Referencias}

Angulo, L. (2014). Prácticas financieras riesgosas para afrontar la crisis económica en los hogares: entre malabarismos con el dinero y sobreendeudamien- 
to. Desacatos, 44, 51-66. Recuperado de http:// www.scielo.org. $\mathrm{mx} / \mathrm{scielo}$.php?pid=S1607050X2014000100005\&script=sci_abstract .

Blázquez, M. y Budría, S. (2015). Income deprivation and mental well-being: The role of non-cognitive skills. Economics and Human Biology, 17, 16-28. doi:10.1016/j.ehb.2014.11.004

Clayton, M., Liñares-Zegarra, J. y Wilson, J.O.S.

(2015). Does debt affect health? Cross country evidence on the debt-health nexus. Journal Social Science \& Medicine, 130, 51-58. doi:10.1016/j.socscimed.2015.02.002

Comisión Nacional Bancaria y de Valores. (2017) Reporte Nacional de inclusión financiera 8. Comisión Nacional Bancaria y de Valores. Recuperado de http://www.cnbv.gob.mx/Inclusi\%C3\%B3n/Documents/Reportes\%20de\%20IF/Reporte\%20de \%20Inclusion\%20Financiera\%208.pdf

Danes, S. N. (1994). Parental percentions of children's financial socialization. Financial Counseling and Planning, 5, 127-146. Recuperado de https:// afcpe.org/assets/pdf/vol-58.pdf

Delval, J. (2007). Aspectos de la construcción del conocimiento sobre la sociedad. Educar, Curitiba, 30, 45-64. Recuperado de https://www.scielo.br/pdf/ er/n30/a04n30

Denegri, M., Del Valle, C., González, Y., Etchebarne, S., Mieres, M., Sandoval, D., Chávez, D. y Ojeda, X. (2013). Educación económica y financiera para la formación inicial de profesores: herramientas conceptuales y didácticas. Temuco: Ediciones Universidad de La Frontera. Recuperado de http://huma- 
nidades.ufro.cl/images/libros/Educacion_Economica_y financieracompressed.pdf

Denegri, M., Araneda, K., Ceppi, P., Olave, N., Olivares,

P. y Sepúlveda, J. (2016). Alfabetización económi-

ca y actitudes hacia la compra en universitarios posterior a un programa de educación económica. Revista de Estudios y Experiencias en Educación, 15(29), 65-81. doi:10.21703/rexe.20162965814

Denegri, M., Caro, C., Cerda, M. I., Eschmann, K., Martínez, N. y Sepúlveda, J. (2017). Relación entre actitudes hacia el endeudamiento y discrepancia del yo en estudiantes de pedagogía chilenos. Actualidades Investigativas en Educación, 17(3), 106-134. doi:10.15517/aie.v17i3.29248

Denegri, M., Sepúlveda, J. y Layera, F. (2018). Comprender la economía: Educación económica y financiera en la infancia desde una perspectiva constructivista. Ensino em Re-Vista, 25(1), 57-81. doi:10.14393ER-v25n1a2018-03

Diez-Martínez, E. (2009). La alfabetización socioeconómica y financiera y la educación para el consumo sostenible en México: algunas reflexiones desde la psicología y la educación. CPU-e, Revista de Investigación Educativa, 8. Recuperado de http:// www.uv.mx/cpue/num8/opinion/alfabetizacion.html

Donnelly, G., Iyerb, R. y Howell, R. (2012). The Big Five personality traits, material values, and financial well-being of self-described money managers. Journal of Economic Psychology. 33, 1129-1142. doi:10.1016/j.joep.2012.08.001

Downing, J. (2016). The health effects of the foreclosure crisis and unaffordable housing: A systematic re- 
view and explanation of evidence. Social Science \& Medicine, 162, 88-96. doi:10.1016/ j.socscimed.2016.06.014

Dunn, L.F. y Mirzaie, I.A. (2016). Consumer debt stress, changes in household debt, and the great recession. Economic Inquiry, 54(1), 201-214. doi: https://doi.org/10.1111/ecin.12218

Durante, K., \& Laran, J. (2016) The Effect of Stress on Consumer Saving and Spending. Journal of Marketing Research, 53(5), 814-828. doi:10.1509/ jmr.15.0319

Esquivel, L. (2017, 3 de abril). Cartera vencida de 12\%; la más alta registrada en 3 años por la alza en tasas. La Razón. Recuperado de https://www.razon.com.mx/negocios/cartera-vencida-de-12-la-masalta-en-3-anos-por-alza-en-tasas/

Falconi, A., Gemmill, A., Karasek, D., Goodman, J., Anderson, B., Lee, M., Bellows, B. \& Catalano, R. (2016). Stroke-attributable death among older persons during the great recession. Economics and Human Biology, 21, 56-63. doi:10.1016/ j.ehb.2015.11.005

García, F. (2017, 17 de noviembre). Buen fin no es endeudamiento para las familias. Milenio. Recuperado de http://www.milenio.com/negocios/buen-finno-es-endeudamiento-para-las-familias

Guth, W., Wärneryd, K. E. y Lea, S. E. G. (1992). Economic psychology and experimental economics. Journal of Economic Psychology, 13(2) 199-201. Hernández. A. (2017, 6 de agosto). Aumentan deudas con tarjetas de crédito. El universal. Recuperado de http://www.eluniversal.com.mx/articulo/nacion/ 
sociedad/2017/08/6/aumentan-deudas-con-tarjetas-de-credito

Hinojosa, G. (2008). El tratamiento estadístico de las redes semánticas naturales. Revista Internacional de Ciencias Sociales y Humanidades SOCIOTAM, 16(1), 133-154. Recuperado de: http://www.redalyc.org/articulo.oa?id=65411190007

Katona, G. (1951). Análisis psicológico del comportamiento económico. Buenos Aires: El Ateneo. Katona, G. y Mueller, E. (1995). The dynamics of consumer reactions. Nueva York: New York University Press.

Lozano, C. y Fuentes, F. (2011). Análisis cualitativo de la toma de decisiones de ahorro o endeudamiento en periodos de crisis económica. Revista investigación operacional, 32(1), 53-66. Recuperado de https://dialnet.unirioja.es/descarga/articulo/ 3682792.pdf

Lusardi, A \& Tufano, P. (2015). Debt Literacy, Financial Experiences, and Overindebtedness. Journal of Pension Economics and Finance, 14(4), 1-41 Mansilla, L., Denegri, M. y Álvarez, B. (2016). Relación entre actitudes hacia el endeudamiento y locus de control del consumidor en estudiantes universitarios. Suma Psicológica, 23(1), 1-9. doi:10.1016/j.sumpsi.2015.11.002

Quintanilla, I. (2010). La psicología económica y del consumidor en la sociedad de la complejidad y la incertidumbre. Información psicológica, 100, 115128. Recuperado de https://dialnet.unirioja.es/servlet/articulo?codigo $=3642857$ 
Reis, F. I., Mendes, K. y Grigion, A. C. (2015). Causas e consequências da dívida no cartão de crédito: uma análise multifatores. Revista de Administração São Paulo, 50(2), 169-182. doi:10.5700/ rausp1192

Reyes, I. (1993). Las redes semánticas naturales, su conceptualización y su utilización en la construcción de instrumentos. Revista de Psicología Social y Personalidad, 9(1), 81-97.

Shen, S., Sam, A. G. y Jones, E. (2014). Credit Card Indebtedness and Psychological Well-Being Over Time: Empirical Evidence from a Household Survey. Journal of Consumer Affairs, 48(3), 431-456. doi:10.1111/joca.12047

Valdez, J. L. (1996). La evaluación del auto concepto a través de la técnica de redes semánticas. Revista Mexicana de Psicología, 13(2), 175-185.

Van Raaij, W. F (1981). Economic Psychology. Journal of Economic Psychology, 1, 1-24. doi:10.1016/0167-4870(81)90002-7

Van Raaij, W. F. (1999). Economic Psychology Between Psychology and Economics: An Introduction. Applied Psychology: An International Review, 48(3), 263-272. doi:10.1111/j.14640597.1999.tb00001.x 


\section{(c) (1) (2) (2)}

\section{Esta obra está bajo una \\ Licencia Creative Commons Atribución-NoComercial-Compar- tirlgual 4.0 Internacional}

Usted es libre de compartir o adaptar el material en cualquier medio o formato bajo las condiciones siguientes: (a) debe reconocer adecuadamente la autoría, proporcionar un enlace a la licencia e indicar si se han realizado cambios; (b) no puede utilizar el material para una finalidad comercial y (c) si remezcla, transforma o crea a partir del material, deberá difundir sus contribuciones bajo la misma licencia que el original.

Resumen de la licencia https://creativecommons.org/licenses/by-nc-sa/4.0/deed.es_ES

Texto completo de la licencia https://creativecommons.org/licenses/by-nc-sa/4.0/legalcode 